\title{
Discriminating Fingerprint Images of Other Images
}

\author{
Armando Rodríguez-Fonte and José Hernández-Palancar \\ Advanced Technologies Application Center (CENATAV), Cuba \\ $\{$ afonte, jpalancar\}@cenatav.co.cu
}

\begin{abstract}
In this paper we propose several features which describe a fingerprint image. These features are based on orientation field and ridges characteristics. We introduce an algorithm to decide if an image is a fingerprint or not. This algorithm is not a fake fingerprint detector, it is oriented to discriminate between fingerprints images and other images. The classification accuracy was tested using a set of databases of fingerprints and non-fingerprints images (like common objects images, face images and iris images). For 2000 fingerprint images our approach shows its robustness achieving a $99.65 \%$ of True Positive Rate (TPR). Carrying out an experimentation for 3525 non-fingerprint images, our approach achieved $97.47 \%$ of reject.
\end{abstract}

\section{Introduction}

Fingerprints as a kind of human biometrics have been widely used for people recognition in commercial and forensic areas due to its uniqueness and immutability [1. Its applications have gone beyond the criminal issues to a mean of assuring individual rights. Former fingerprint analysis techniques (highly reliable contextual analysis) oriented to identification have evolved around off-line applications (large database searches, high capacity computers) related to law enforcement operations. On the other hand, newer security uses of automatic fingerprint identification systems (AFIS) involving verification, such as banking, shopping or access control, require real-time operations with low cost. For these cases it is important to develop fast fingerprint analysis techniques that still retain the characteristics of the fingerprint image [1].

An Automatic Fingerprint Identification System (AFIS) can receive nonfingerprint images. If these images are not detected automatically in the enrollment stage, the feature extraction algorithm could detect some minutiae and the accuracy in several verification tasks may be seriously affected. Also nonfingerprints images may cause several inconsistencies in enrollment algorithms because they might not be prepared to receive these image types. As a first stage in the AFIS, it is necessary ensure that the received image is a fingerprint with appropriate quality for matching. There are some tools to evaluate the quality of fingerprint image like [2] but those tools are not reliable to detect non-fingerprint images [3. In a scenario like a multi-biometric platform, due to human or system errors, during the enrollment process could be received non-fingerprint images

E. Bayro-Corrochano and E. Hancock (Eds.): CIARP 2014, LNCS 8827, pp. 524531 2014.

(C) Springer International Publishing Switzerland 2014 
as blank images, face images, iris images, etc, instead of fingerprint images. We can help to improve the data consistency of the system avoiding to storage nonfingerprint images and so to improve the identification accuracy.

In the literature there are many approaches for fingerprint classification: singular points based, structure based, frequency based, mathematical model based and hybrid methods. A fingerprint could be classified as rolled or plain [4]. But the problem of identification if an image is really a fingerprint had not been attacked before 2013. Recently Yoon and Jain [3] proposed a model to distinguish fingerprint images of non-fingerprint images and altered fingerprints by measuring the abnormality in the orientation field of the image. They presented a global orientation field model of fingerprint represented in terms of ordinary differential equations. They used Support Vector Machines (SVMs) classifiers to compute a measure of fingerprintness and then classify the image. The algorithm [3] has good results for tested databases but the implementation is not very easy because they tried to resolve the problem of orientation field estimation using a mathematical model and as a second result they created a fingerprint pattern. Our algorithm is simple because our only problem is identify fingerprint images of other images. Our ideas are very clear and the accuracy is very high.

The rest of the paper is organized as follow: section 2 describes some important aspects of fingerprint like orientation, coherence and ridges characteristics. Six classification features are defined in section [3. One classification algorithm is proposed in section 4. Section 5 shows the results of experimental tests and finally section 6] comments conclusions and future work.

\section{Orientation, Coherence and Ridges Characteristics in Fingerprint Images}

The local orientation of a ridge at a pixel $(\mathrm{x}, \mathrm{y})$ is the angle $\Theta_{x y}$ which is formed by the horizontal axis and the fingerprint ridges, crossing through an arbitrary neighborhood centered at (x, y) [1]. First introduced by Grasselli [5], the fingerprint orientation image, is a matrix whose elements encode the local orientation of fingerprint ridges. Each element $\Theta_{i j}$ corresponding to the node (i, j) of a square-meshed grid located over the pixel $\left(x_{i}, y_{j}\right)$, denotes the average orientation of the fingerprint ridges in a neighborhood of $\left(x_{i}, y_{i}\right)$ [1].

One way for extracting local ridge orientation is based on computation of gradients in the fingerprint image $([6],[7])$. The gradient $\nabla(x, y)$ at point $[\mathrm{x}$, $\mathrm{y}]$ of image, is a two-dimensional vector $\left[\nabla_{x}(x, y), \nabla_{y}(x, y)\right]$, where $\nabla_{x}$ and $\nabla_{y}$ components are the derivatives of image at $[\mathrm{x}, \mathrm{y}]$ with respect to the $\mathrm{x}$ and $\mathrm{y}$ directions, respectively. These methods compute the dominant $\theta_{i j}$ by combining multiple gradient estimates within a $17 \times 17$ window $W$ centered at $\left[x_{i}, y_{i}\right]$ :

$$
G_{x y}=\sum_{h=-8}^{8} \sum_{k=-8}^{8} \nabla_{x}\left(x_{i}+h, y_{j}+k\right) * \nabla_{y}\left(x_{i}+h, y_{j}+k\right),
$$




$$
\begin{aligned}
& G_{x x}=\sum_{h=-8}^{8} \sum_{k=-8}^{8} \nabla_{x}\left(x_{i}+h, y_{j}+k\right)^{2}, \\
& G_{y y}=\sum_{h=-8}^{8} \sum_{k=-8}^{8} \nabla_{y}\left(x_{i}+h, y_{j}+k\right)^{2}, \\
& \theta_{i j}=\frac{\Pi}{2}+\frac{1}{2} \operatorname{atan} 2\left(2 G_{x y}, G_{x x}-G_{y y}\right) .
\end{aligned}
$$

The reliability $r$ of the orientation can be derived by the coherence of the orientation vectors in the local window $W([6]$, [7]). In fact, due to the continuity and smoothness of fingerprint ridges, sharp orientation changes often indicate unreliable estimation. Using gradient method approach, the coherence could be computed as follows:

$$
r_{i j}=\operatorname{coherence}\left(\theta_{i j}\right)=\frac{\sqrt{\left(G_{x x}-G_{y y}\right)^{2}+4 G_{x y}^{2}}}{G_{x x}+G_{y y}}
$$

We modified orientation image using coherence and we defined an orient-coh image (see figure 1). Pixels of orientation image where the reliability is lower than a threshold are considerate bad pixels and their values in orient-coh image will be change to white. The rest of the pixels are called good pixels and they will conserve their value in orient-coh image (see equation [6). Bad pixels in orient-coh image tend to correspond to low quality zones of fingerprint because the ridges pattern and the orientation field could be affected in those zones (see figure $1(\mathrm{c})$.

$$
\text { Orient }-\operatorname{coh}(x, y)= \begin{cases}255 & \text { if } r_{x y} \leq \text { threshold } \\ \text { orientation }(x, y) & \text { otherwise }\end{cases}
$$

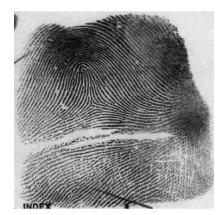

(a)

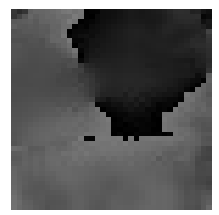

(b)

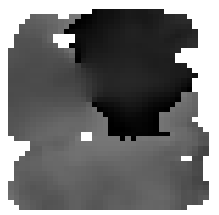

(c)

Fig. 1. Fingerprint with low reliability zones in orientation image (b). These zones contains several bad pixels. These pixels are white in orient-coh image (c). The image (a) is classified by the algorithm as fingerprints.

An important step in the most of the algorithms for minutiae extraction is binarization step. The objective of this step is to remark ridges over valleys in fingerprint images. All fingerprints have ridges and valleys. These are distinctive elements for these images types. After binarization step, a feature extraction algorithm can detect minutiae for fingerprint image. When the binarization step 
is applied to a non-fingerprint image, several structures like ridges could appear and the extraction algorithm may detect minutiae for those (see figures $2(\mathrm{~b}), 2(\mathrm{f})$ and $2(\mathrm{j})$. But in the almost all cases this pattern of ridges is very different to fingerprint pattern. We observed two features related with ridges very useful to help to discriminate fingerprint from other images types: average width of ridges and horizontal count of ridges. The average width of ridges for fingerprints is about 5 to 7 pixels [8]. The width of ridges can be calculated using the method proposed by [9]. The number of ridges in the horizontal is computed starting in the center of image and moving horizontally to both sides.

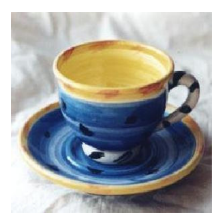

(a)

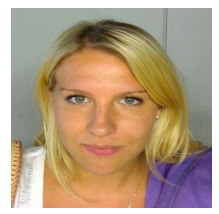

(e)

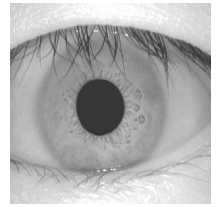

(i)

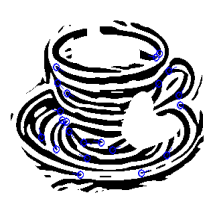

(b)

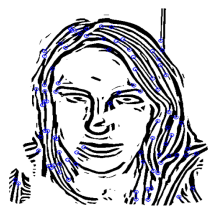

(f)

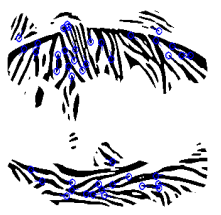

(j)

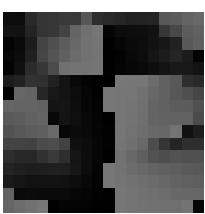

(c)

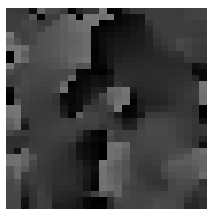

(g)

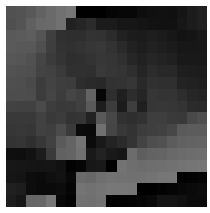

(k)

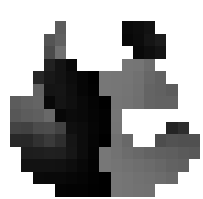

(d)

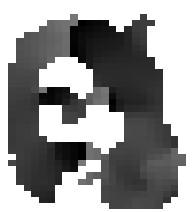

(h)

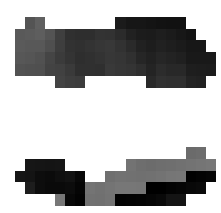

(l)

Fig. 2. Several examples of non-fingerprint images. Original images (a), (e) and (i). Binarized images with minutiae (b), (f) and (j). Orientation images (c), (g) and (k). Orient-coh images (d), (h) and (l).

\section{Features for Fingerprint Classification}

Our classification algorithm is based on the analysis of orient-coh image and two ridge characteristics. In images with very low quality the number of bad pixels in orient-coh image grows and the probability to be classified as non-fingerprint is higher. We expect a high number of bad pixels in non-fingerprint orient-coh images because the reliability of their orientation image tends to be very lower than fingerprint images with medium or high quality. Now we will define four features to describe a fingerprint based on this idea. 


\section{Definition of Good Block (Feature 1)}

A good block in orient-coh image is a block where there is at least one good pixel (see figure 3(a) . In most of cases the number of good blocks in a fingerprint orient-coh image is high because there is a small number of bad pixels. The block size used in this algorithm was 16.

\section{Definition of Average Pixel Degree (Feature 2)}

The degree of a pixel (PD) is defined as:

$$
P D=8-B P N
$$

BPN is the number of bad pixels neighbors to it (see figure 3(b)) . Commonly the average of pixel degree in a fingerprint orient-coh image is close to 8 .

\section{Definition of Maximum Connected Component (Feature 3)}

A set of good pixels linked by a relation of neighborhood in orient-coh image is denoted as connected component. We define maximum connected component as the set with highest cardinality (see figure $3(\mathrm{c})$ ). The number of pixels in the maximum connected component of a fingerprint orient-coh image should be close to the total of pixels in that image.

Definition of Number of Bad Pixels in the Center Blocks (Feature 4) Another feature is the number of bad pixels in the center blocks of the orient-coh images (see figure $3(\mathrm{~d})$ ). This zone of fingerprint is very important for matching methods because many minutiae and singular points are located there [10. We consider that a fingerprint must have a very small number of bad pixels near to center of orient-coh image.

Features 5 and Feature $\mathbf{6}$ are the average width of ridges and the horizontal count of ridges respectively.

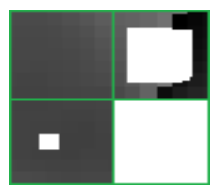

(a)

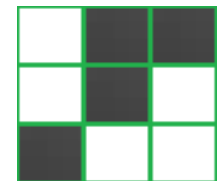

(b)

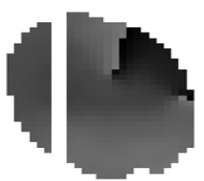

(c)

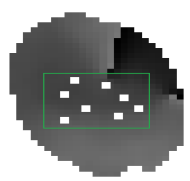

(d)

Fig. 3. Feature examples for orient-coh images. (a) Four blocks in orient-coh image, three of those are good, but the bottom-right is not good. (b) Nine pixels of orient-coh image, the degree of the center pixel is 3. (c) Orient-coh image with two connected components, the right connected component is the maximum connected component in the image. (d) Orient-Coh image with 8 bad pixels in the center blocks.

Support Vector Machines (SVMs) are a set of supervised learning methods used for classification, regression and other learning tasks. SVMs are classifiers that model the decision boundary between the classes as separated hyperplanes. We used SVMs for binary classification. Often example data is not li-nearly separable and SVMs introduce the notion of a kernel induced feature space 
moving data to a higher dimensional space where the details are separable. The kernel type used was radial basis function [11. SVMs are intuitive, theoretically well-founded, and they has been successful in practical applications.

\section{Fingerprint Image Classification Algorithm}

Figure 4 show a flow chart of the classification algorithm. It receives an image to classify it as fingerprint or non-fingerprint. First and second steps are to get orient-coh image and binarized image respectively. Then it builds a 6 -dimensional vector with the normalized values for each feature seen previously. After that, the SVMs classifier is applied to obtain a classification value. Finally this value is returned as a boolean decision.

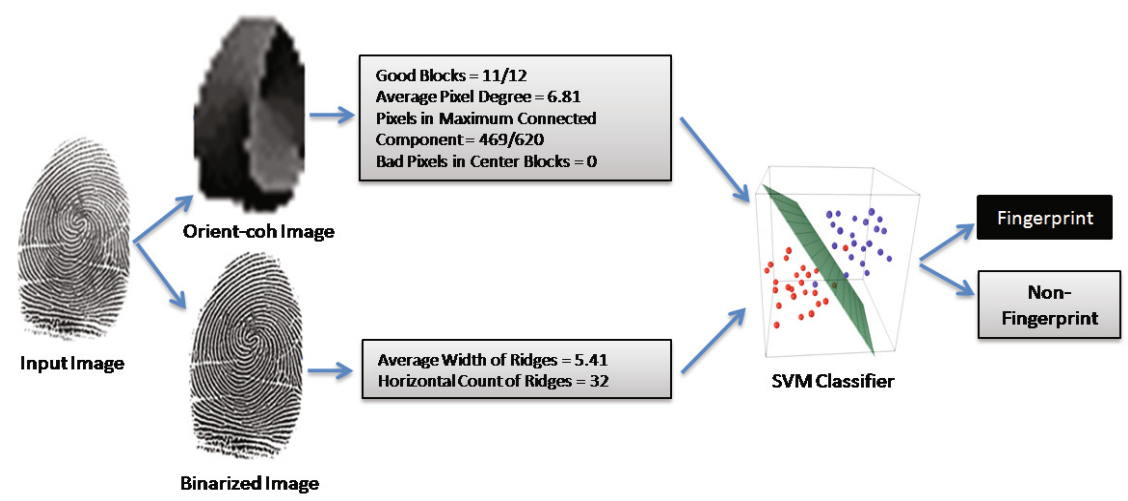

Fig. 4. Fingerprint image classification algorithm flow chart

\section{Experimental Results}

Our experiments were carried out using a personal computer (64 bits) Intel (R) Core (TM) 2 Quad CPU Q9450 @ 2.66 GHz and 4 Gb of RAM. The algorithm for fingerprint classification was implement in $\mathrm{C}++$ language and compiled using $\mathrm{g}++$ compiler of GNU/Linux.

The training of SVMs classifier was done using 400 fingerprint impressions from Nist SD14 12 and 400 non-fingerprint images (object images, iris images and face images randomly taken). These images were excluded from the analysis of the classification accuracy.

We compared the proposed algorithm against Yoon and Jain algorithm [3]. They tested 2000 fingerprint images from Nist SD4 [13] and 2000 object images randomly taken from Imagesnet [14. They used 2000 face images from Feret Database [15] and 2000 iris images from MBGC Database [16. We took 2000 fingerprint images from Nist SD4 and 1247 objects images from Imagenet (that is because our version of this database only has 1247 images). Feret and MBGC are 
not available for us at the moment so we took 1521 face images from Bio-ID-Face 17] and 757 iris images from Casia-v1 [18. The table 1 shows the accuracy of our proposal and Yoon and Jain. Our approach was better for fingerprint images, object images and iris images. Their proposal was better for faces images. Figure 5 shows the Receiver Operating Characteristic (ROC) curve for the performance of the classification algorithm. Our approach achieved a $99 \%$ of TPR at $1 \%$ of FPR, while the method proposed by Yoon and Jain achieved a $90 \%$ of TPR for the same percent of FPR. In this case our proposal is better than [3] in nine percent values.

Table 1. Comparison between proposed algorithm and Yoon and Jain [3] algorithm

\begin{tabular}{|c|c|c|c|c|}
\hline Algorithm & Nist SD4 & ImageNet & Face & Iris \\
\hline Yoon and Jain 3 & $98.65 \%$ & $91.55 \%$ & $99.20 \%$ & $97.70 \%$ \\
\hline Proposed & $99.65 \%$ & $93.02 \%$ & $99.0 \%$ & $100 \%$ \\
\hline
\end{tabular}

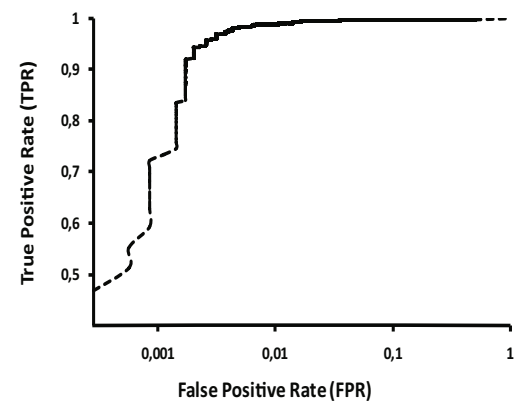

Fig. 5. ROC curve for fingerprint vs. non-fingerprint (objects, faces and iris) classification

\section{Conclusions and Future Work}

We have proposed an algorithm to discriminate fingerprint images of other images types. The percent of correct classification for 2000 fingerprint images was $99.65 \%$. For 3525 non-fingerprint images our approach achieved $97.47 \%$ of reject. These results show that our approach achieves high accuracy in classification tasks, outperforming the other state-of-the-art work. We did not use any preprocessing and the computational complexity for feature extraction is low.

Future work on classification fingerprint includes:

1. Analysis of other features minutiae-based. We did not include minutiae information in the algorithm but we think those features can help to improve its performance.

2. We consider the configuration of singular points as one of the significant aspects to enrich the proposed fingerprint pattern because these points are very remarkable in fingerprints. 


\section{References}

1. Maltoni, D., Maio, D., Jain, A.K., Prabhakar, S.: Handbook of Fingerprint Recognition, 2nd edn. Springer (2009)

2. Tabassi, E., Wilson, C., Watson, C.: Fingerprint Image Quality. NISTIR 7151 (August 2004)

3. Yoon, S., Anil, K.: Jain Is There a Fingerprint Pattern in the Image. In: Proceedings of the 6th IAPR International Conference on Biometrics (ICB 2013), Madrid, June 4-7 (2013)

4. Nadgir, R., Ross, A.: Roll versus Plain Prints: An Experimental Study Using the NIST SD 29 Database (2006)

5. Grasselli, A.: On the automatic classification of fingerprints. In: Watanabe, S. (ed.) Methodologies of Pattern Recognition, Academic, New York (1969)

6. M. Kass, A.P. Witkin. Analyzing oriented patterns. Computer Vision, Graphics, and Image Processing, 37 3, 362-385 (1987)

7. Bazen, A.M., Gerez, S.H.: Systematic Methods for the Computation of the Directional Fields and Singular Points of Fingerprints. IEEE Trans. Pattern Anal. Mach. Intell. 24(7), 905-919 (2002)

8. Wu, C., Zhou, J., Bian, Z., Rong, G.: Robust Crease Detection in Fingerprint Images. In: IEEE Computer Society Conference on Computer Vision and Pattern Recognition, CVPR, June 16-22, IEEE Computer Society, Madison (2003) ISBN: 0-7695-1900-8

9. Cheng, J., Tian, J.: Fingerprint enhancement with dyadic scale-space. Pattern Recognition Letters 25(11), 1273-1284 (2004)

10. Kim, J.K., Chae, S.-H., Lim, S.J., Pan, S.B., Moon, D.: Performance Analysis of Hybrid Fingerprint Matching Methods. In: The Second International Conference on Future Generation Communication and Networking, FGCN 2008, Hainan Island, China. Workshops, vol. 2 (2008)

11. Chang, C.-C., Lin, C.-J.: LIBSVM: a library for support vector machines (2001)

12. NIST Special Database 14, Mated Fingerprint Card Pairs 2

13. NIST Special Database 4: NIST 8-Bit Gray Scale Images of Fingerprint Image Groups (FIGS)

14. Deng, J., Dong, W., Socher, R., Li, L.-J., Li, K., Fei, L.: Imagenet: A Large-Scale Hierarchical Image Database. In: Proc. CVPR, pp. 248-255 (2009)

15. The Facial Recognition Technology (FERET) Database

16. Multiple Biometric Grand Challenge (MBGC) Database

17. BioID Technology Research. The BioID Face Database (2001), http://www.bioid.com

18. CASIA Iris image database (version 1.0), Institute of automation (IA), Chinese Academy of Sciences, Beijing, China (2007),

http://www.cbsr.ia.ac.cn/IrisDatabase.htm 